\title{
Simulation Analysis of Cable Coupling Effect based on CST
}

\author{
Shuanshuan FENG*, Zengrui LI, Jianxun SU \\ School of Information \&Engineering \\ Communication University of China \\ Beijing, China 10024 \\ *E-mail: 2605941755@qq.com \\ $+*$ Corresponding author
}

\begin{abstract}
In view of the cavity in the complex electromagnetic compatibility of the problems of cable layout, based on the theory of transmission, network theory to establish a single cable and cavity cable model in CST cable studio ,and the circuit model was established in CST cable studio (CST-DS).The coupling interference value between the cables is analyzed by simulation. The size of coupling crosstalk and its influencing factors were analyzed based on the simulation results.
\end{abstract}

Keywords-cable; crosstalk; Coupling; transmission line theory

\section{INTRODUCTION}

Due to the rapid development of the modern electronic information age, system of electromagnetic compatibility problems become more and more prominent, the system devices, cable, cable and equipment such as various coupling interference phenomena exist everywhere. The analysis of the problem of cable crosstalk is a large part in the analysis of system - level emc.

Crosstalk is a near field coupling problem, indicating the interference of the adjacent signal path to a signal path, which is the signal coupling in one line to the other[1]. It can describe the coupling of intentional signal or noise to a signal path. Crosstalk between adjacent cables can be caused by the electric field generated by the mutual capacitance coupling, namely a wire on the energy through the coupling capacitance coupling between the two wire to another wire[2]; It can also be caused by the coupling of magnetic field generated by the mutual inductance, which can be analyzed by the mutual inductance of two circuits.

How to reduce the influence of cable due to the coupling of the surrounding cable, the premise is to establish the electromagnetic coupling simulation model of the cable, and to estimate the coupling effect of the target cable through simulation calculation. However, due to the calculation process is very complicated, the traditional research model receive cable type relatively single, such as c. r. Paul [3] research of the frequency domain response of unshielded transmission line cable, Bellan [4], analysis and calculation of non-uniform beam cable coupling effect.
This paper establish a single cable and cavity cable model in CST cable studio

\section{BASIC THEORY OF ELECTROMAGNETIC COUPLING}

There are two forms of capacitive coupling and inductance coupling in the induction field of the cable, the most easily capacitance coupling in high impedance high frequency circuits[5]. Two circuits of the cable, when they get close and the existence of potential difference, the cable of the electric field in a circuit to another cable is induced in the circuit, and vice versa, the two phase interaction, make them mutual influence of electric field change, this is called to electric field coupling. Two cables to electric field coupling degree depends on the shape, size and location of the cable and the properties of the surrounding medium. in RF circuit wire cable, the wire on the coupling interference can be transmitted to all other wires.

In the adjacent space of the current-carrying cable, the magnetic field will be present. If the current varies with time, the magnetic field will change over time, and the changing magnetic field will interfere with other components. The main factors that interfere with the size of the coupling are the frequency of the current, the distance between the two cables, and the length of the common walking line in parallel. In the case of the disturbance source voltage, the disturbance circuit impedance is smaller, the disturbance current is large, the magnetic field is also large, so the inductance coupling is stronger.

\section{DESIGN OF CROSSTALK SIMULATION OF CST CABLE STUDIO}

\section{A. Two Single String Crosstalk}

According to CST cable studio point, period, the order of the path to build out of the cable channels and cavity model, start the $2 \mathrm{D}$ (TL) modeling to extract the spice model, and design studio established circuit connection, created the time domain and frequency domain simulation tasks, the following figure 1 shows the specific size of the model. 


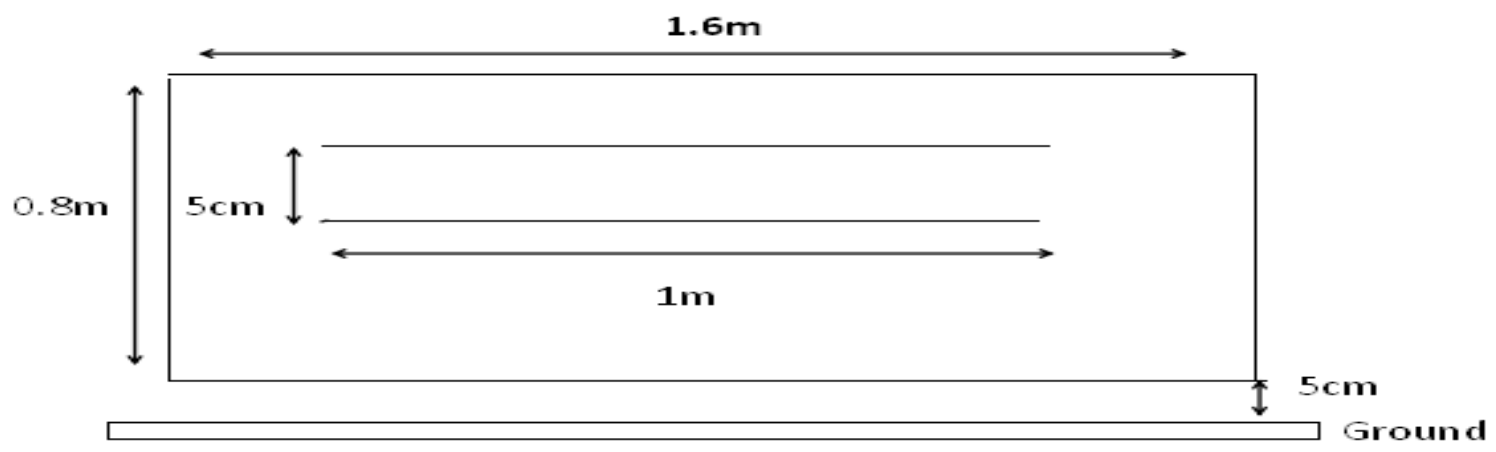

Figure 1. Geometry dimensions of the model

As shown in figure 1: the metal plate size is $1.6 \mathrm{~m} *$ than the metal plate $5 \mathrm{~cm}$. The distance between the two $0.8 \mathrm{~m}$, the cable length is $1 \mathrm{~m}$, and the wire beam is higher cables is $5 \mathrm{~cm}$.

Figure 2. Cable model established in CST (cs)

As shown in figure 2: the grey background is a metal plate with no thickness. The yellow pellet is the node of the four ports, and the blue line is the linear form defined by the CST cable studio.

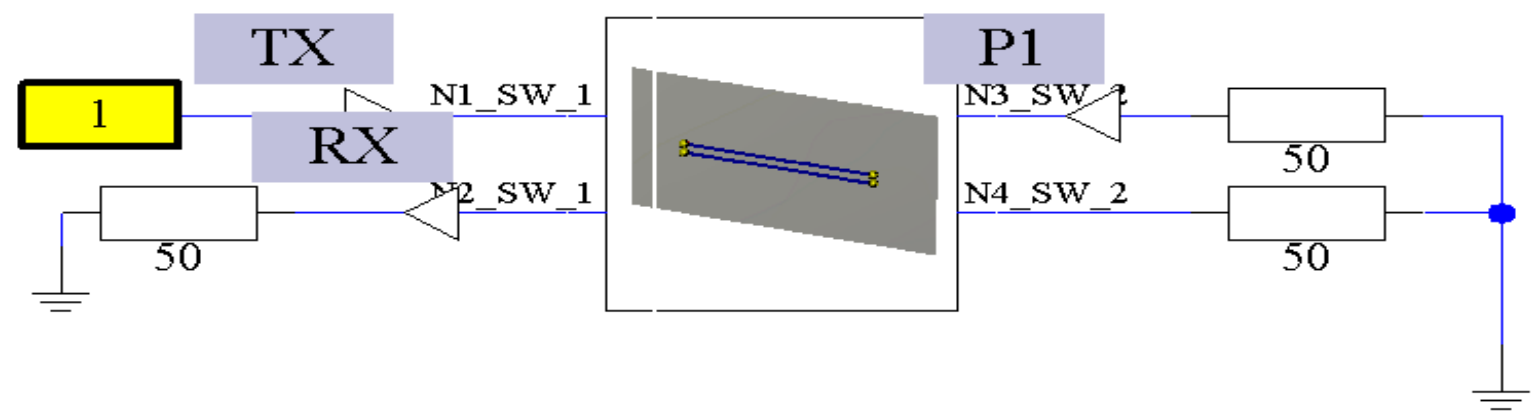

Figure 3.

Model of load circuit established in CST (DS)

As shown in figure 3: The simulation of cable is required to connect the cables through the design studio in CST, cable 1 port as excitation source need to access a port, $50 \mathrm{ohm}$ resistance for ports in the cable, then grounding. The RX and TX probes are set to see the simulation results.

\section{B. Analysis of Simulation Result}

The simulation excitation selects the sweep source with the amplitude of $0 \sim 200 \mathrm{M}$ in the full spectrum is $1 \mathrm{~V}$, and port 1 as the input port of the excitation square wave signal. The port 1 input excitation source is an actual square wave signal, as shown in figure 4 . 


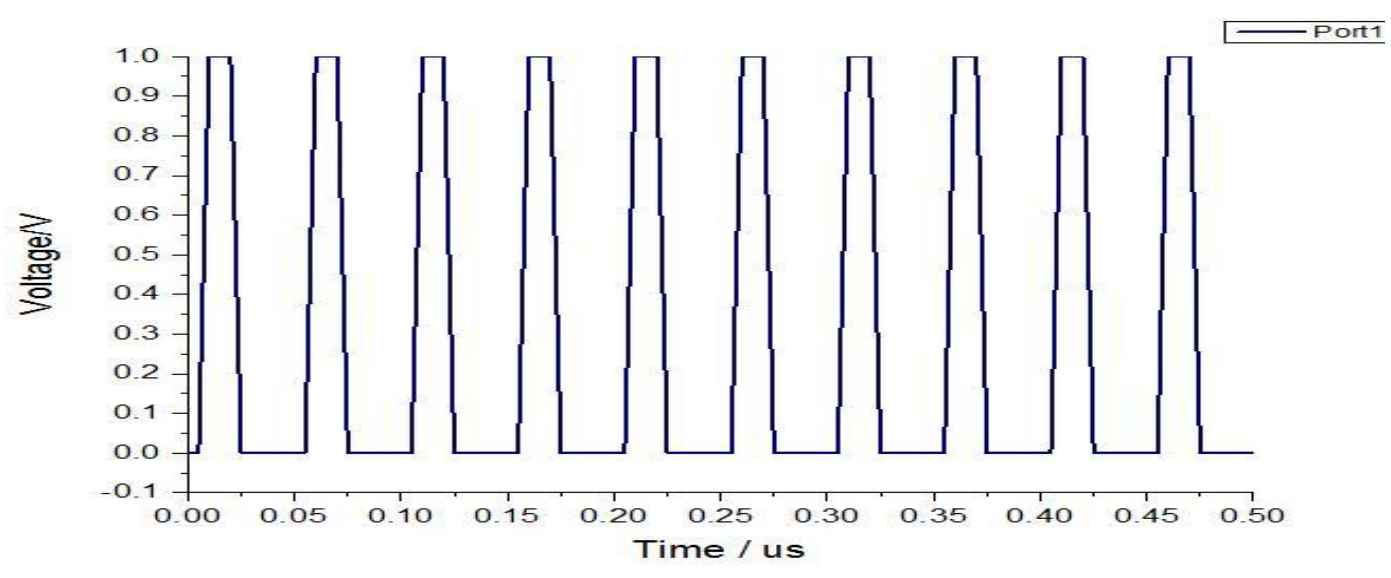

Figure $4 . \quad$ Square wave signal

As shown in figure 4: the excitation source is a square wave signal of $0 \sim 1 \mathrm{~V}$, working time of $0.50 \mathrm{us}$, and the jump interval is 0.25 us.

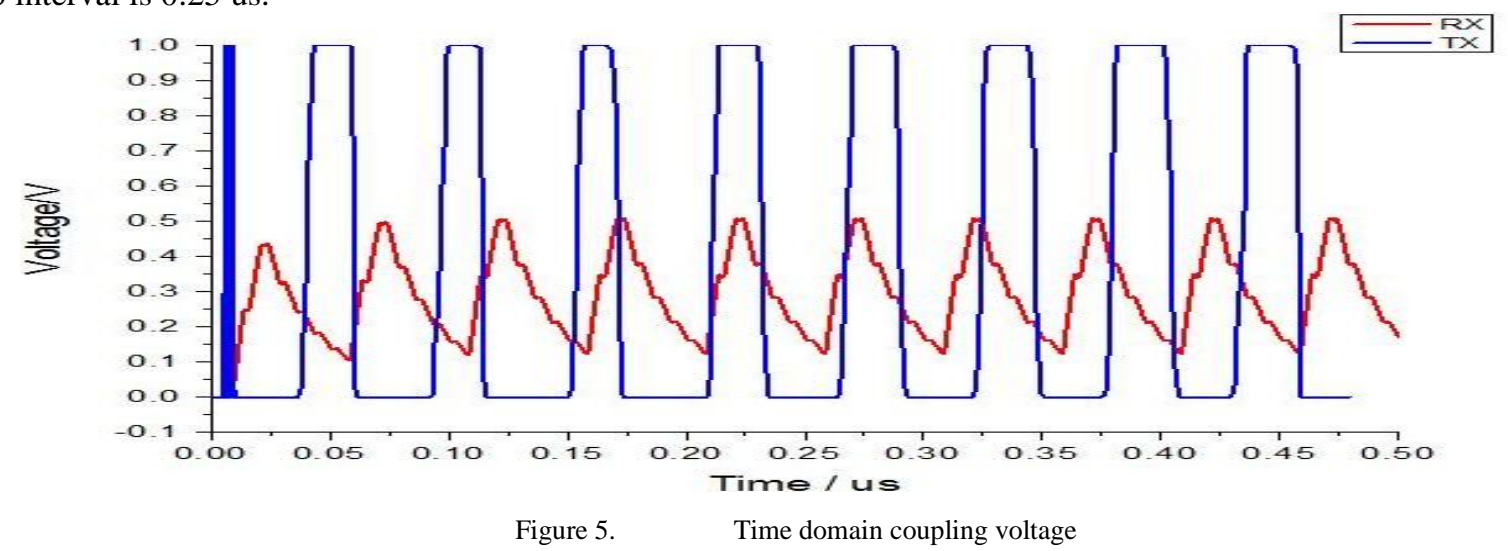

As shown in figure 5: Port RX is highly coupled with the excitation signal, and the crosstalk voltage is proportional to the excitation signal.

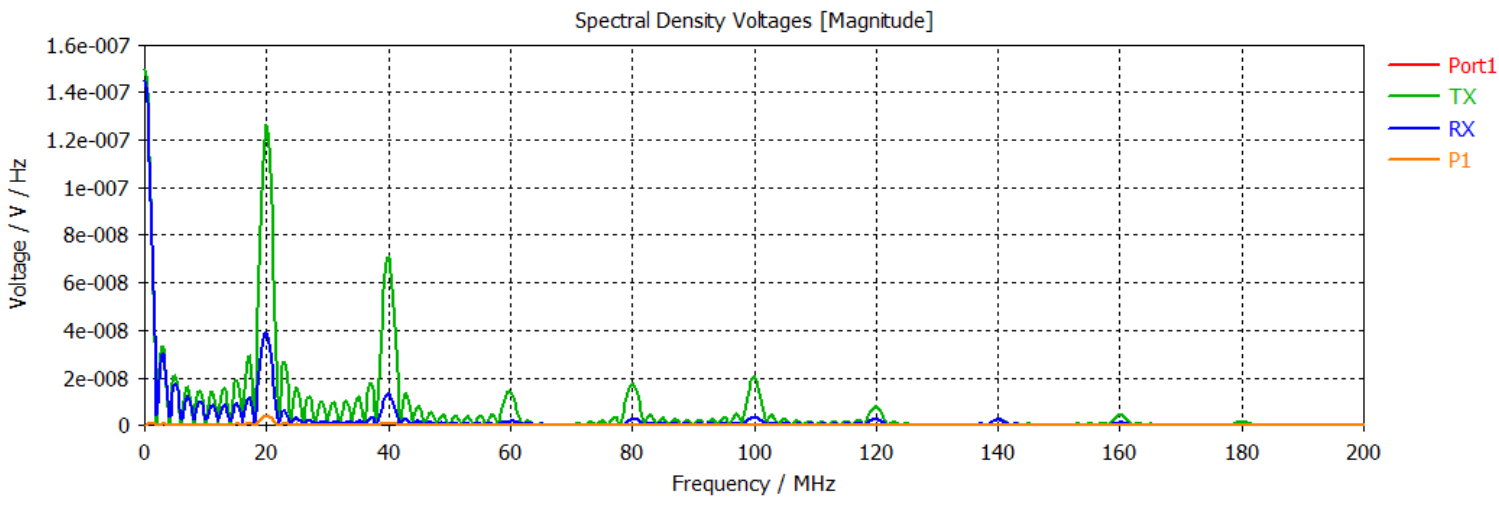

Figure 6. Frequency domain coupling voltage

As shown in figure 6: the voltage amplitude of port TX and RX is basically the same, and at low frequency stage the voltage value is higher, and the voltage value decreases as the frequency increases.

\section{Single Cable Cavity Crosstalk}

The following figure 7 shows the specific size of the model and cable relationship with the relative position of cavity. 


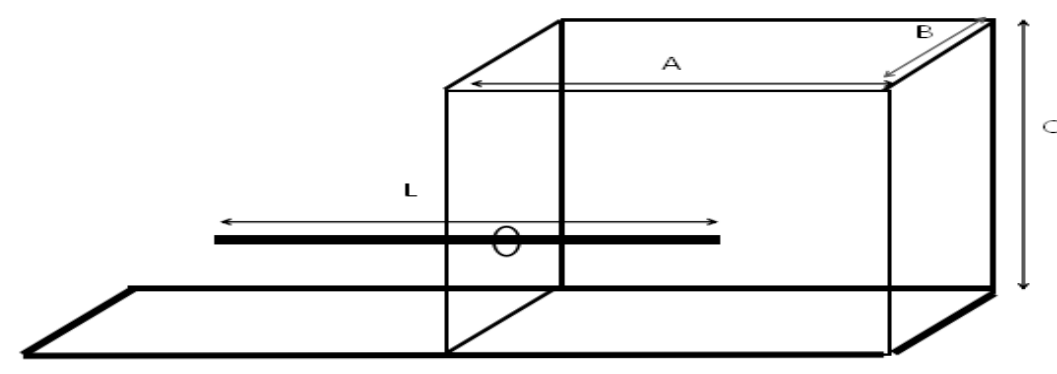

Figure 7. Geometry dimensions of the model

As shown in figure 7: The size of the metal floor is $200 \mathrm{~cm} * 200 \mathrm{~cm}$, and the cavity is directly placed on the floor in the size of $A=200 \mathrm{~cm}, B=200 \mathrm{~cm}, C=200 \mathrm{~cm}$, the

diameter of the circular hole in the side of the cavity is $1 \mathrm{~cm}$, and the length of the cable through the hole is $\mathrm{L}=200 \mathrm{~cm}$.

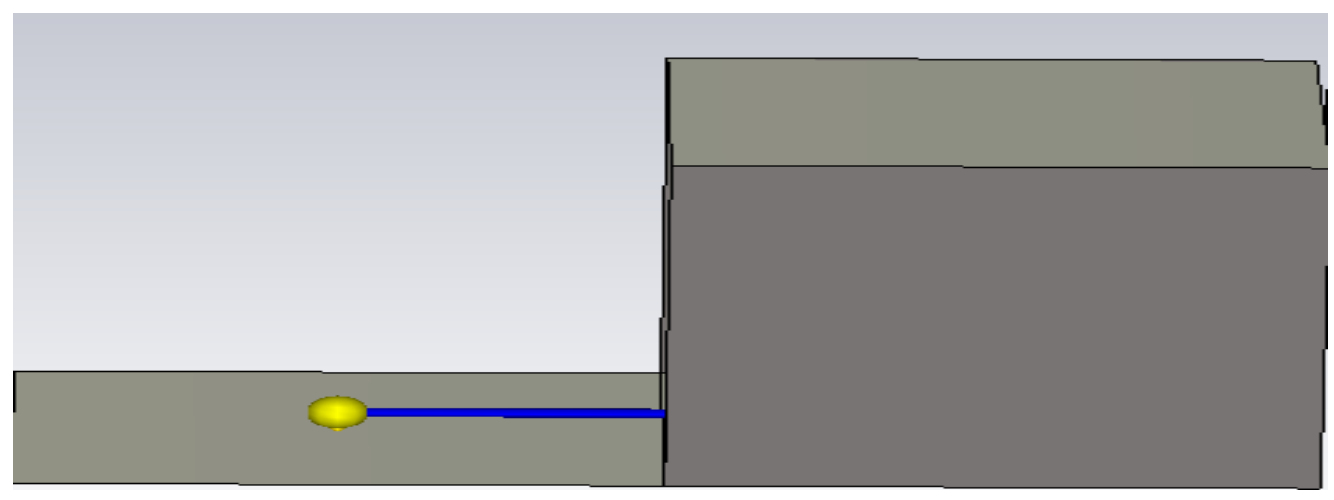

Figure 8. Single cable model established in CST (cs)

As shown in figure 8: gray background bottom surface is a not the thickness of the metal plate, a cube is cavity shell, yellow balls as the node of the cable port, blue thread is defined using CST studio cable (cavity internal have half cable).

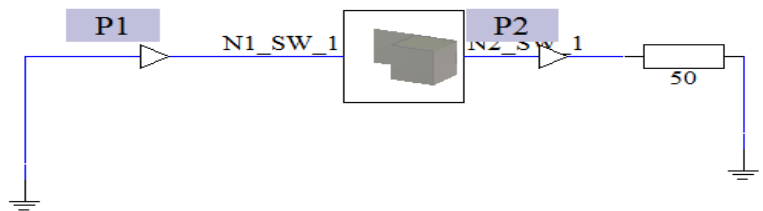

Figure 9.
As shown in figure 9: the cable simulation in need to connect all ports of cable design by CST cable studio, the port impedance according to the actual situation, then grounding. The $\mathrm{P} 1$ and $\mathrm{P} 2$ probes are set to see the simulation results.

\section{Analysis Of Simulation Result}

EMP irradiation simulation of the cavity model is used to set EMP excitation signal and plane wave irradiation in CST cable studio. The excitation signal and the plane wave type used are shown in figure 10.

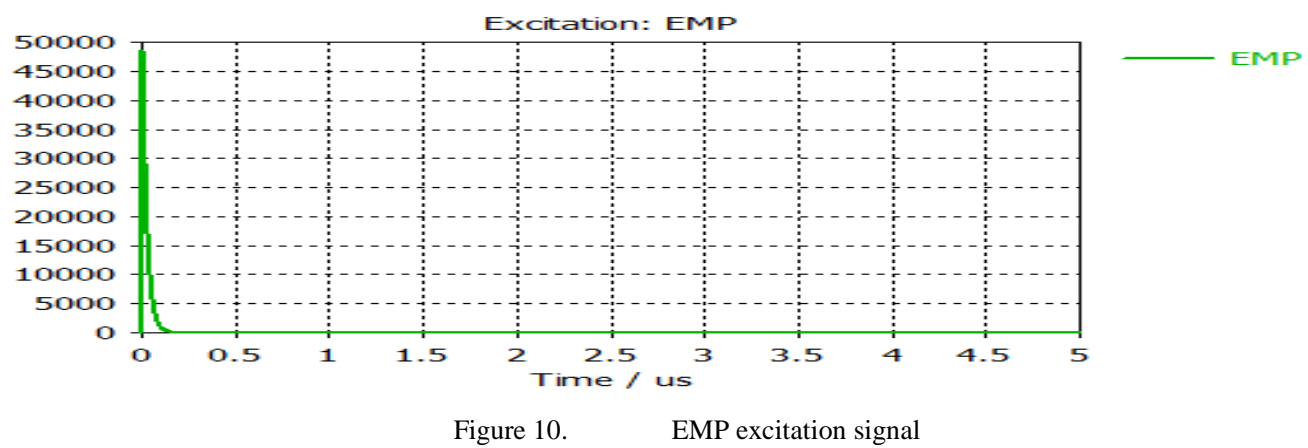

As shown in figure 10: the excitation signal is only stimulated in the first few seconds, and the intensity of excitation decreases gradually over time from the beginning of the high intensity, and finally disappears completely at about $\mathrm{t}=0.2 \mathrm{us}$. 


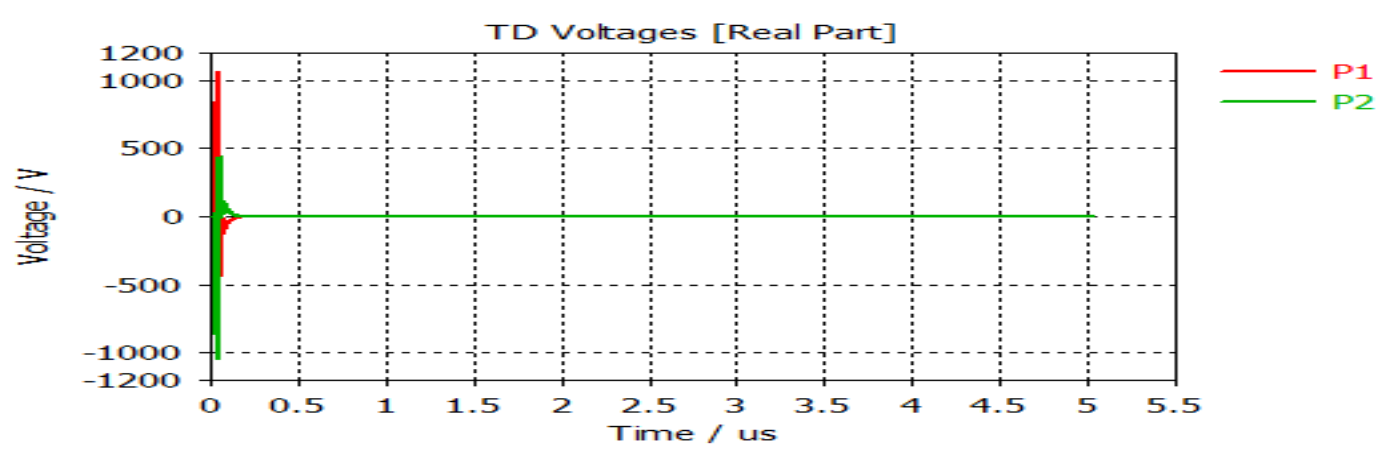

Figure 11. Time domain coupling voltage

As shown in figure 11: the voltage at port P1 and P2 moves approximately the same. After $\mathrm{t}=0.2 \mathrm{us}$, the voltage starts to fluctuate violently at $\mathrm{t}=0.1 \mathrm{us}$, and the waveform is reduced to $0 \mathrm{~V}$.

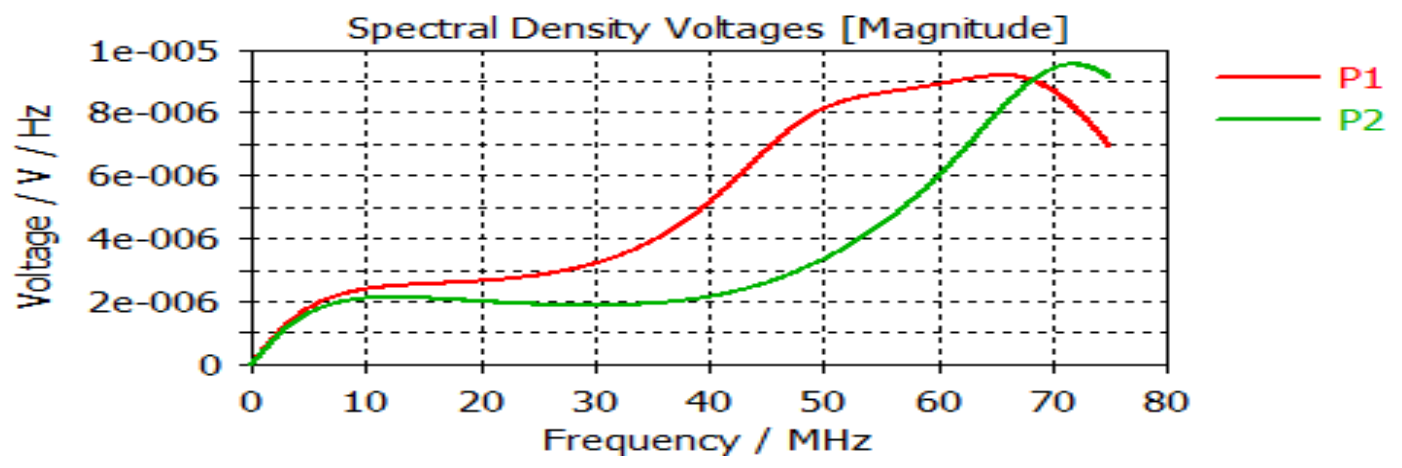

Figure 12. Frequency domain coupling voltage

As shown in figure 12: port $\mathrm{P} 1, \mathrm{P} 2$ in the same direction of the spectral density, the voltage basic trend with the increase of the frequency increases gradually, the voltage has peak until $\mathrm{f}=$ about $68 \mathrm{HZ}$, then there is a little down.

\section{SUMMARY}

The above simulation briefly analyses the crosstalk coupling problems of the cables, and the crosstalk coupling results of the cavity cable are obtained through the CST simulation. The results show that the problem of the coupling of the cavity cable is closely related to its working time, frequency size and incentive signal.

For the above reasons, the solutions to the anti-interference of cables are mainly grounded, shielded, filtered and so on, so the selection of cable lines is of particular importance. This requires that in selecting the type of cable, electromagnetic compatibility should not be ignored except for the circuit characteristics and environmental conditions. In terms of electromagnetic compatibility, the factors that need to be considered include the waveform of transmission signal, frequency range, circuit sensitivity, electromagnetic environment and so on. The results of this study can be used to analyze the shielding effectiveness of the perforated lumen.

\section{REFERENCES}

[1] PAUL $\mathrm{C}$ R. Introduction to electromagnetic compatibility[ M].Hoboken, NJ: Wiley, 2006.

[2] PAUL C R. MCKNIGHT J A: Prediction of crosstalk invo- lving twisted pairs of wires, Part I : A transmission line model for twisted wire pairs [ J ] . IEEE Trans. Electromagn. Compat. EMC-21, $92-105$ ( 1979 ).
[3] Weiguo $\mathrm{Wu}$. The vibration and noise control of high speed boats [J]. Journal of wu han university of technology and technology, 2000, 24 (1) : 16-19.

[4] BIOT M A The theory of propagation of elastic waves in a fluid-saturated porous solid I Low frequency range J Aocust soc Am 1956,28:168-178.

[5] Weston, [Canada] David a. Yang Zi you king three translation, electric magnetic compatibility with the original principle and application of Beijing: mechanical industry publishing club, 2006. 